\title{
The Clinical Manifestation Variety and Management Choice of Meckel's Diverticulum with Complication: A Single Center Experience
}

\author{
Tingliang Fu $\mathbb{D}^{\mathbb{D}},{ }^{1}$ Xiaoliang Xu, ${ }^{1}$ Lei Geng $\mathbb{D},{ }^{1}$ Yanli Huang $\mathbb{D},{ }^{2}$ Guojian Ding, ${ }^{1}$ and Hong $\mathrm{Ji}^{3}$ \\ ${ }^{1}$ Department of Pediatric Surgery, Binzhou Medical University Hospital, Binzhou, Shandong 256603, China \\ ${ }^{2}$ Department of Surgery, Jinan Maternity and Child Care Hospital Affiliated to Shandong First Medical University, 3rd Jian-Guo \\ Xiaojing Road, Jinan, Shandong 250000, China \\ ${ }^{3}$ Department of Pathology, Binzhou Medical University Hospital, Binzhou, Shandong 256603, China
}

Correspondence should be addressed to Lei Geng; drglei@163.com and Yanli Huang; hyl-2987@163.com

Received 14 December 2020; Revised 9 January 2021; Accepted 23 January 2021; Published 9 February 2021

Academic Editor: Roberto Caronna

Copyright (C) 2021 Tingliang Fu et al. This is an open access article distributed under the Creative Commons Attribution License, which permits unrestricted use, distribution, and reproduction in any medium, provided the original work is properly cited.

Background. The study was to analyze the clinical manifestation variety and management choices of symptomatic Meckel's diverticulum in children. Methods. From July 2008 to October 2018, 28 cases of Meckel's diverticulum with a variety of complications were admitted to our hospital. The clinical data included age, gender, symptoms and signs, investigations, intraoperative and pathological findings, and outcome. Results. The ratio of males to females was $2.5: 1$. The diagnoses were made by ${ }^{99} \mathrm{~m}$ Tc-pertechnetate scan (in 5 cases) and by exploratory laparotomy (in 2 cases). The initial diagnosis in the other cases includes intussusception (in 4 cases), acute appendicitis (in 5 cases), intestinal obstruction (unknown origin), peritonitis, and even shock in 12 cases. Laparoscopic surgery was performed in 8 cases; 18 cases underwent open surgery. Excision of partial bowel segment with diverticulum and primary anastomosis was done in 22 cases and wedge resection of diverticulum in 4 cases. Two other cases received nonoperative therapy and went to other hospitals to receive surgical management. Ectopic gastric mucosa in the diverticulum was found in 9 cases, including 6 cases with lower gastrointestinal bleeding. Conclusion. The clinical characteristics of Meckel's diverticulum varied. Children with hematochezia, peritonitis, and intestinal obstruction without history of prior abdominal operation should be suspected with this disease until proven otherwise. Hematochezia is often associated with ectopic gastric mucosa in the diverticulum. Laparoscopic surgery should be one of the choices for the diagnosis and treatment of Meckel's diverticulum with complications.

\section{Introduction}

Meckel's diverticulum is a congenital malformation caused by incomplete degeneration of the yolk sac in the early embryo, and the incidence is about $1.4 \%$ to $2 \%$ of the population as a whole [1]. But only about $2 \%$ of the population with Meckel's diverticulum present symptoms [2]. It can complicate with lower gastrointestinal bleeding, different types of intestinal obstruction, diverticulitis or perforation, and carcinoid or adenocarcinoma [3]. Intestinal obstruction is a more common complication in adults, whereas in children, bleeding is the more common complication [4]. It is key to make early diagnosis and give prompt and appropriate treatment for children presenting acute abdomen associated with Meckel's diverticulum. Any delayed diagnosis and treatment may lead to serious consequences, even life threatening. The present observation was to analyze the clinical data and experience of Meckel's diverticulum in a single medical center and to emphasize its variety of clinical manifestations and rational management choices so as to improve the clinical outcome of patients with this unusual disorder.

\section{Methods}

From July 2008 to October 2018, 28 cases with Meckel's diverticulum were admitted to our hospital. The diagnosis 


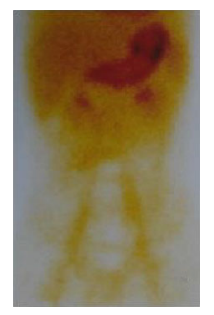

(a)

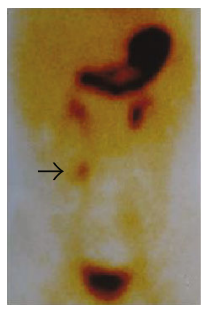

(c)

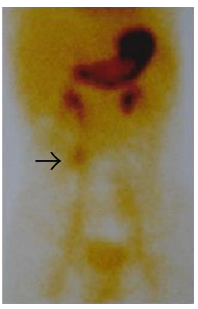

(b)

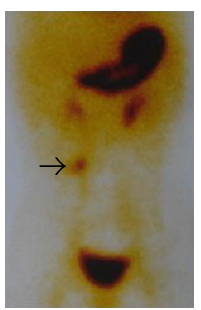

(d)

Figure 1: A 12-year-old boy with massive lower gastrointestinal bleeding; ${ }^{99 \mathrm{~m}}$ Tc-pertechnetate scan was positive. The arrows show the existence of ectopic gastric mucosa.

was confirmed by ${ }^{99 \mathrm{~m}}$ Tc-pertechnetate scan, ultrasound, surgical exploration, and histopathological examination. The clinical data included age, gender, symptoms, investigation, surgical procedure, and clinical outcome. The existence of ectopic gastric mucosa was evaluated in the resected diverticulum specimen.

\section{Results}

3.1. Clinical Manifestations. The ratio of males to females was $2.5: 1$. The clinical manifestations of symptomatic Meckel's diverticulum included lower gastrointestinal bleeding, intussusception, intestinal volvulus, abdominal internal hernia, adhesive bowel obstruction, diverticulitis, perforation, shock, etc. There were 8 cases with lower gastrointestinal bleeding, and five were diagnosed by ${ }^{99 \mathrm{~m}} \mathrm{Tc}$-pertechnetate scan (Figure 1).

There were 11 cases with intestinal obstruction, including intussusception in 4 , entrapment or internal hernia in 6 , and bowel necrosis due to volvulus in one case. Six cases with peritonitis due to diverticulitis or perforation were diagnosed by laparotomy.

3.2. Surgical Procedure and Clinical Outcome. Laparoscopic exploration was performed in 8 cases. Once the diagnosis was made, a semicircle incision around the umbilicus was done to exposure the diverticulum and to excise it with an adjacent bowel segment. Open surgery was performed in 18 cases, including cuneiform resection or bowel segment excision and primary anastomosis with good outcome.

3.3. Pathological Findings. In our case series, 9/19 (47.37\%) cases with ectopic gastric mucosa in the diverticulum were detected by pathological examination, including 6/8 (75\%) cases with intestinal bleeding (Figure 2). Two other cases with diverticulitis or gastrointestinal bleeding were diagnosed by ultrasound and transferred to other hospitals to

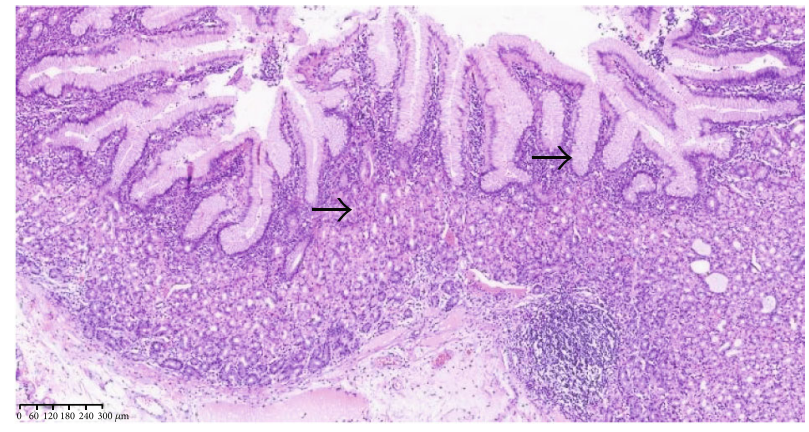

Figure 2: Ectopic gastric mucosa was found in Meckel's diverticulum; the arrows show the ectopic gastric mucosa.

undergo surgical management. Ectopic gastric mucosa could not be identified due to necrotic diverticulum or other reasons in 7 cases.

\section{Discussion}

4.1. Gender and Age Distribution. Lee et al. [5] reported that the ratio of males to females who suffered from Meckel's diverticulum associated with complications is about $2.3: 1$, while Rho et al. [6] reported that the ratio is $7.5: 1$. In the present study, the ratio was 2.5:1. Mackey and Dineen [7] reported that there is no gender difference in the population with asymptomatic Meckel's diverticulum. In general, the incidence of Meckel's diverticulum associated with complication is more common in males than in females [8]. The high peak of the occurrence is patients under 5 years of age [9]. Other studies reported that $66.5 \%-75 \%$ of the patients are under 10 years of age $[6,8,10]$. In our case series, $79 \%$ of symptomatic cases were younger than 10 years of age.

4.2. Pathological Features. The incidence of ectopic gastric tissue in symptomatic Meckel's diverticulum is $45 \% \sim 80 \%$ 
[11]. The length of Meckel's diverticulum and the location of the ectopic gastric mucosa play an important role in surgical management $[12,13]$. In symptomatic Meckel's diverticulum, ectopic gastric mucosa usually locates in the basal part, so diverticulectomy without an associated bowel segment may be insufficient. The ectopic gastric mucosa can be found in different parts of the diverticulum or scattered in small islands [14]. Ectopic pancreatic tissue is usually located at the top of the diverticulum and is yellowish-white, granular, so it is easy to identify [15]. In our case series, 9/19 (47.37\%) cases with ectopic gastric mucosa were detected by pathological examination, especially in patients with lower gastrointestinal bleeding $(75 \%)$.

4.3. The Many Faces of Clinical Representations. The variety of clinical manifestations in patients with symptomatic Meckel's diverticulum including small intestinal obstruction, intussusception, inflammation, lower gastrointestinal hemorrhage, perforation, and other rare conditions was reported $[4,9,16$, 17]. Ectopic gastric mucosa is present in $90 \%$ of cases of Meckel's diverticulum with hemorrhage. In our case series, ectopic gastric mucosa was detected in $75 \%$ of the patients with lower gastrointestinal bleeding, while those without gastrointestinal hemorrhage, only $16.7 \%$. Diverticulum with an abnormal cord causes intestinal volvulus, internal hernia, intussusception, and other rare disorders, such as twisted intestine, presenting small bowel obstruction without history of prior abdominal surgery. It is usually difficult to make preoperative diagnosis of inflammation of Meckel's diverticulum [18]. From the present group of cases, lack of awareness and the development of nonspecific symptoms may make the diagnosis of Meckel's diverticulum difficult. Especially in patients with unexplanatory shock, an acute abdomen should be suspected until proven otherwise. In a word, early and correct clinical diagnosis is based on a high index of suspicion [19].

4.4. Surgical Treatment Choice. In the era of minimally invasive surgery, laparoscopic diverticulectomy for Meckel's diverticulum is one of the choices. But, in the case with short and wide basal diverticulum, laparoscopic resection and anastomosis could become difficult. Onen et al. [10] suggested that if the base of diverticulum is too large, or if there is an ulcer on the mesentery side, the diverticulum and adjacent ileum segment should be excised and primary end-toend anastomosis should be performed. At present, laparoscopic exploration should be one of the preferred approaches for the diagnosis and management of Meckel's diverticulum $[11,20]$. However, it is difficult to determine whether there is a mass or thick base in the diverticulum and to evaluate the presence of ectopic gastric mucosa, so it is easier for surgeons to choose laparoscopy-assisted surgery [21]. Gezer et al. [17] found that touching the tissue under laparoscopy can mislead the existence or absence of ectopic gastric mucosa. In the present case series, precise diagnosis before operation was not made in 8 cases with Meckel's diverticulum associated with complications. The diagnosis of Meckel's diverticulum was made by laparoscopic exploration. Meckel's diverticulum cuneiform resection or Meckel's diverticu- lum plus adjacent segment resection with primary intestinal anastomosis was undergone with good outcome.

In conclusion, patients with Meckel's diverticulum may present variable symptoms and pathological findings. In children, hematochezia or intestinal obstruction without history of prior abdominal operation should be considered in the differential diagnosis of Meckel's diverticulum. Meckel's diverticulum complicated with lower gastrointestinal bleeding is usually related to the existence of ectopic gastric mucosa. ${ }^{99 \mathrm{~m}} \mathrm{Tc}$-pertechnetate scan should be selected for the differential diagnosis of Meckel's diverticulum with lower gastrointestinal bleeding. Laparoscopic exploration and management should be one of the choices.

\section{Data Availability}

The data used to support the findings of the retrospective analysis could be available from the corresponding authors upon request.

\section{Conflicts of Interest}

All authors have no conflict of interest to declare.

\section{Authors' Contributions}

Tingliang Fu, Lei Geng, and Yanli Huang performed the conception and design. Yanli Huang, Xiaoliang Xu, Hong Ji, and Guojian Ding performed the clinical and pathological data collection. Yanli Huang contributed to the data analysis and interpretation of the paper. Yanli Huang, Lei Geng, and Tingliang Fu contributed to the manuscript writing and approved this paper for submission. All authors have approved the final version of this manuscript.

\section{Acknowledgments}

We thank all the sick children and our colleagues for their contribution. This work was supported by the Key Scientific and Research Plan Fund from Binzhou Medical University (BY2012KJZD02) in Yantai, China.

\section{References}

[1] C. C. Hansen and K. Søreide, "Systematic review of epidemiology, presentation, and management of Meckel's diverticulum in the 21st century," Medicine(Baltimore), vol. 97, no. 35, article e12154, 2018.

[2] C. C. Huang, M. W. Lai, F. M. Hwang et al., "Diverse presentations in pediatric Meckel's diverticulum: a review of 100 cases," Pediatrics and Neonatology, vol. 55, no. 5, pp. 369375, 2014.

[3] M. Kapischke, K. Bley, and E. Deltz, "Meckel's diverticulum-a disease associated with a colored clinical picture," Surgical Endoscopy, vol. 17, no. 2, p. 351, 2003.

[4] R. Kassir, T. Debs, C. Boutet et al., "Intussusception of the Meckel's diverticulum within its own lumen: unknown complication," International Journal of Surgery Case Reports, vol. 10, pp. 111-114, 2015. 
[5] K. H. Lee, C. K. Yeung, Y. H. Tam, W. T. Ng, and K. F. Yip, "Laparoscopy for definitive diagnosis and treatment of gastrointestinal bleeding of obscure origin in children," Journal of Pediatric Surgery, vol. 35, no. 9, pp. 1291-1293, 2000.

[6] J. H. Rho, J. S. Kim, S. Y. Kim et al., "Clinical features of symptomatic Meckel's diverticulum in children: comparison of scintigraphic and non-scintigraphic diagnosis," Pediatric Gastroenterology, Hepatology \& Nutrition, vol. 16, no. 1, pp. 41-48, 2013.

[7] W. C. Mackey and P. Dineen, "A fifty year experience with Meckel's diverticulum," Surgery, Gynecology \& Obstetrics, vol. 156, pp. 56-64, 1983.

[8] Y. A. Lee, J. H. Seo, H. S. Youn et al., "Clinical features of symptomatic Meckel's diverticulum," Korean Journal of Pediatric Gastroenterology and Nutrition, vol. 9, no. 2, pp. 193199, 2006.

[9] A. Francis, D. Kantarovich, N. Khoshnam, A. L. Alazraki, B. Patel, and B. M. Shehata, "Pediatric Meckel's diverticulum: report of 208 cases and review of the literature," Fetal and Pediatric Pathology, vol. 35, no. 3, pp. 199-206, 2016.

[10] A. Onen, M. K. Ciğdem, H. Oztürk, S. Otçu, and A. I. Dokucu, "When to resect and when not to resect an asymptomatic Meckel's diverticulum: an ongoing challenge," Pediatric Surgery International, vol. 19, no. 1-2, pp. 57-61, 2003.

[11] J. S. Davis, A. C. Hirzel, M. M. Rodriguez, H. L. Neville, and J. E. Sola, "Heterotopic gastric mucosa mimicking a Meckel's diverticulum in a young girl," Journal of Pediatric Surgery, vol. 50, no. 5, pp. 879-881, 2015.

[12] M. Mukai, H. Takamatsu, H. Noguchi, T. Fukushige, H. Tahara, and T. Kaji, "Does the external appearance of a Meckel's diverticulum assist in choice of the laparoscopic procedure?," Pediatric Surgery International, vol. 18, no. 4, pp. 231-233, 2002.

[13] K. W. Chan, K. H. Lee, J. W. C. Mou, S. T. Cheung, and Y. H. Tam, "Laparoscopic management of complicated Meckel's diverticulum in children: a 10-year review," Surgical Endoscopy, vol. 22, no. 6, pp. 1509-1512, 2008.

[14] J. R. Robinson, H. Correa, A. S. Brinkman, and H. N. Lovvorn 3rd, "Optimizing surgical resection of the bleeding Meckel diverticulum in children," Journal of Pediatric Surgery, vol. 52, no. 10, pp. 1160-1165, 2017.

[15] R. J. Craigie, N. Forrest, S. Nanthakumaran, and A. A. Mahomed, "Laparoscopy in diagnosis and management of Meckel's diverticulum," Journal of Laparoendoscopic \& Advanced Surgical Techniques. Part A, vol. 16, no. 1, pp. 7073, 2006.

[16] L. W. E. van Heurn, M. P. Pakarinen, and T. Wester, "Contemporary management of abdominal surgical emergencies in infants and children," The British Journal of Surgery, vol. 101, no. 1, pp. e24-e33, 2014.

[17] H. Ö. Gezer, A. Temiz, E. İnce, S. S. Ezer, B. Hasbay, and A. Hiçsönmez, "Meckel diverticulum in children: evaluation of macroscopic appearance for guidance in subsequent surgery," Journal of Pediatric Surgery, vol. 51, no. 7, pp. 11771180, 2016.

[18] J. R. Poley, T. E. Thielen, and J. C. Pence, "Bleeding Meckel's diverticulum in a 4-month-old infant: treatment with laparoscopic diverticulectomy. A case report and review of the literature," Clinical and Experimental Gastroenterology, vol. 2, pp. 37-40, 2009.
[19] M. S. Shalaby, K. Kuti, and G. Walker, "Intestinal malrotation and volvulus in infants and children," BMJ, vol. 347, article f6949, 2013.

[20] V. Erol, T. Yoldaş, S. Cin, C. Çalışkan, E. Akgün, and M. Korkut, "Complicated Meckel's diverticulum and therapeutic management," Turkish Journal of Surgery, vol. 29, no. 2, pp. 63-66, 2013.

[21] R. J. Lindeman and K. Søreide, “The many faces of Meckel's diverticulum: update on management in incidental and symptomatic patients," Current Gastroenterology Reports, vol. 22, no. 1 , p. 3,2020 . 